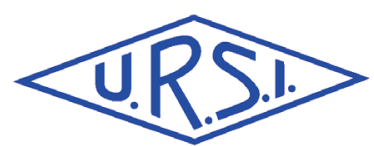

\title{
On the worst case trajectories of microwave links above Belgium
}

\author{
Emmanuel H. Van Lil ${ }^{(1)}$ and Roeland Van Malderen ${ }^{(2)}$ \\ (1) KU Leuven, Heverlee, 3001, Belgium, e-mail: Emmanuel.VanLil@ESAT.KULeuven.Be \\ (2) Royal Meteorological Institute, Uccle 1180, Belgium; e-mail: Roeland.VanMalderen@meteo.be
}

\begin{abstract}
It is common that the national authorities impose large consultation zones around microwave links [1]. When those areas are free of obstacles there is no problem, but usually they are considered as exclusion zones. Those large zones are inspired by the fact that the obstacle for which the authorisation is requested may not be the only one, especially in the case of wind turbines. The national authorities (for Belgium BIPT/IBPT) impose limitations on the projections of the links in a horizontal plane, usually 3 times the largest size of the first Fresnel ellipsoid. In this paper we will investigate the $3 \mathrm{D}$ case to allow more lenient criteria to be applied.
\end{abstract}

\section{Introduction}

The current authorisation criterion implies that a large area is usually excluded from construction of fixed or moving objects like wind turbines. An example of those zones are shown in Fig. 1.

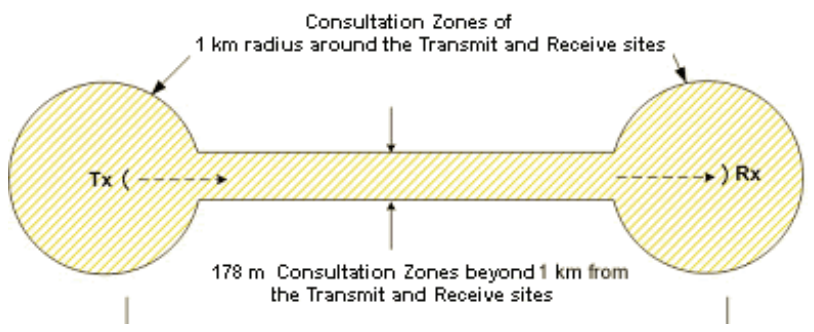

Fig. 1: Consultation zones for a microwave link (from [1])

The trajectory of a microwave link in space is essentially determined by meteorological parameters. The most important are temperature and pressure but also the partial pressure of water vapour has to be taken into account. However, to calculate the trajectory, we also need the derivatives of those parameters with respect to the height above the local ground level.

\section{Basic theory}

Indeed, the refractive index of air is given in the appropriate IUT-R standard [2]:

$$
n=1+77.6 * 10^{-6} / T[p-0.072 e+4810 e / T]
$$

where $T$ is the temperature in degrees Kelvin, $p$ and e the total pressure and the partial pressure of water vapour in $\mathrm{hPa}$. This allows us to follow a wave transmitted over a microwave link if we assume an atmosphere consisting of concentric spheres with a homogeneous refractive index between two consecutive spheres. It has been shown that the equivalent radius of curvature of the path of the wave $R_{e q}$ is given by [3]:

$$
\frac{1}{R_{e q}}=\cos (\psi)\left(\frac{d n}{n d h}+\frac{1}{R_{a}+h}\right)
$$

where $R_{a}$ is the local radius of the earth's surface (smaller than $6378137 \mathrm{~m}$ in the WGS84 ellipsoid) along the direction of the microwave link, $h$ is the altitude above the local ground level, and $\psi$ is the angle of the propagation direction with the tangent plane to the local sphere. Since this angle is very close to $0^{\circ}$, the cosine may be set to 1 (one). For the standard atmosphere, the proportion $K$ between the effective radius to be used to compute the equivalent height above the cord is about $4 / 3$. In fact it lowers the profile of the earth so that the radio horizon is further away than the geometrical one. This is illustrated in Fig. 2 for a $65 \mathrm{~km}$ link on a fictitious terrain with 3 artificial mountains.

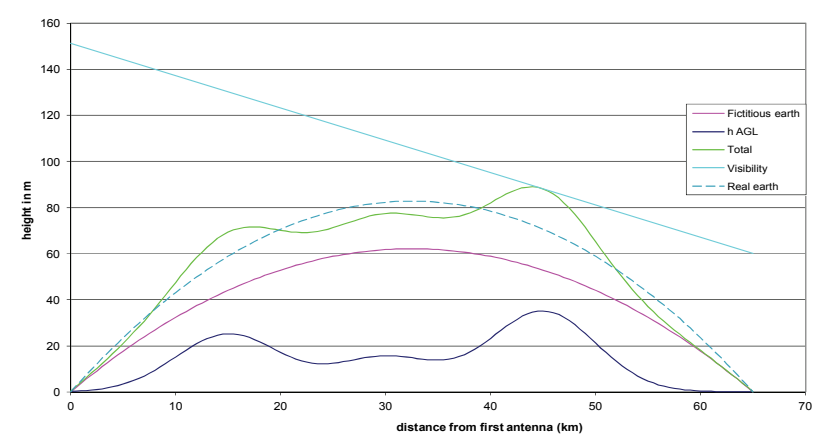

Fig. 2: Fictitious terrain profile with 3 Gaussian mountains at 15,30 and $45 \mathrm{~km}$ of a $65 \mathrm{~km}$ microwave link.

A more realistic profile of a microwave link at $7 \mathrm{GHz}$ with a large wind turbine above the link is shown in Fig. 3. The terrain heights have been obtained from the freely available SRTM (Shuttle Radar Tomography Mission) [4]. The tower is far away from the centre of the link (even if it is not shown on the figure), but, due to the large diameter of the turbine, there is a horizontal overlap with 
the 3 Fresnel radii. A cross-section showing the first ellipsoid and the locus of the tips of the turbine is shown in Fig. 4 for the standard atmosphere. In exceptional propagation circumstances (in this case with a negative $K$ factor) they can become tangent to each other (Fig. 5) and even overlap (values of $-0.1332<K<-0.0014$ ).

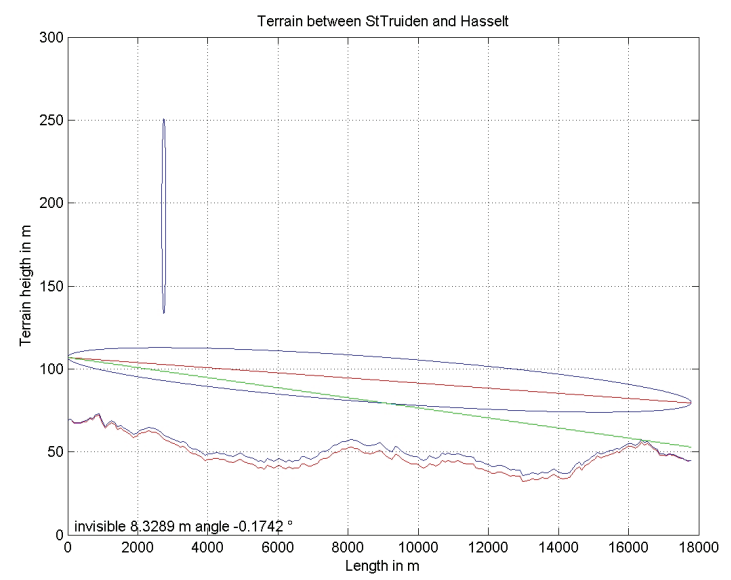

Fig. 3: Real terrain profile of a high wind turbine located above a $7 \mathrm{GHz}$ microwave link of $17.8 \mathrm{~km}$. The red line is the connection between the centres of the antennas and the blue ellipse is the first Fresnel ellipsoid. The terrain without curvature correction is in red, and with correction for the standard atmosphere in blue, as well as the wind turbine. The green line is the radio visibility of the left antenna.

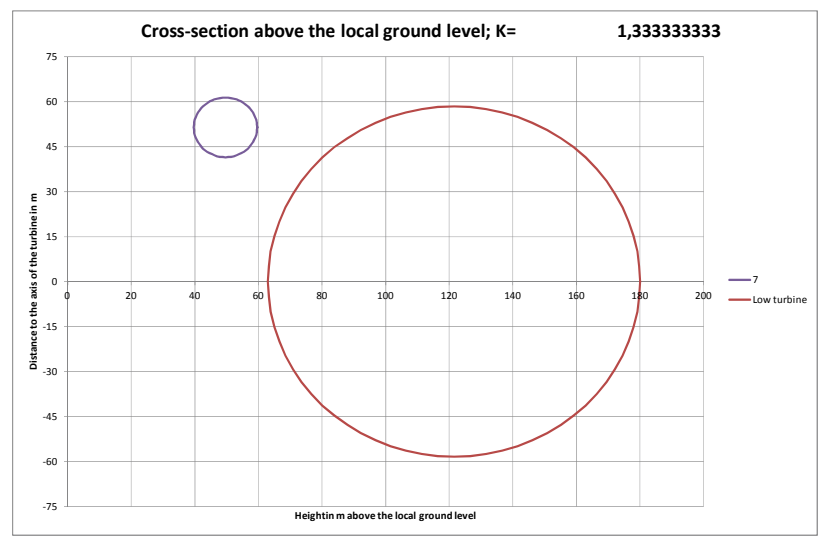

Fig. 4: Cross-section of a high wind turbine located above a $7 \mathrm{GHz}$ microwave link at the location of the turbine for the standard atmosphere with $K=4 / 3$. In this and all next plots, the largest circle is the locus of the tips of the turbine and the smaller ones the cross-sections of the first Fresnel ellipsoids of the links.

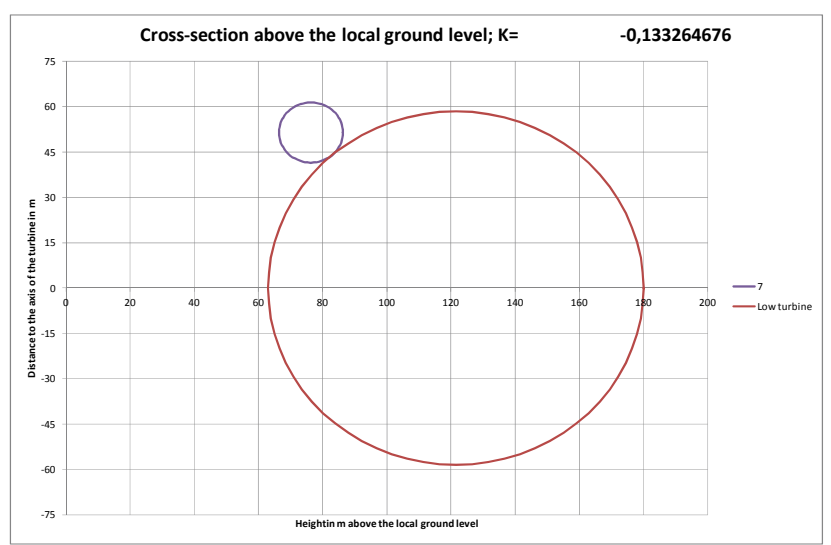

Fig. 5: Cross-section of a high wind turbine located above a $7 \mathrm{GHz}$ microwave link at the location of the turbine for a non-standard atmosphere $(K=-0.133264676)$.

A similar behaviour is valid for two high microwave links above a smaller turbine (at 7 and $12 \mathrm{GHz}$, with antennas at different heights). In Fig. 6, we see that the $12 \mathrm{GHz}$ link is in the worst case position of the turbine blades slightly overlapping with the first Fresnel ellipsoid. The approximate formula from ITU-R [5] does not show any attenuation for this small overlap. The antennas of the 12 $\mathrm{GHz}$ link could be relocated at a higher position. The first Fresnel ellipsoid touches the locus of the tips of the turbine when $K=0.84$, see Fig. 7. At this point, the 12 $\mathrm{GHz}$ microwave link has an attenuation of $0.9 \mathrm{~dB}$.

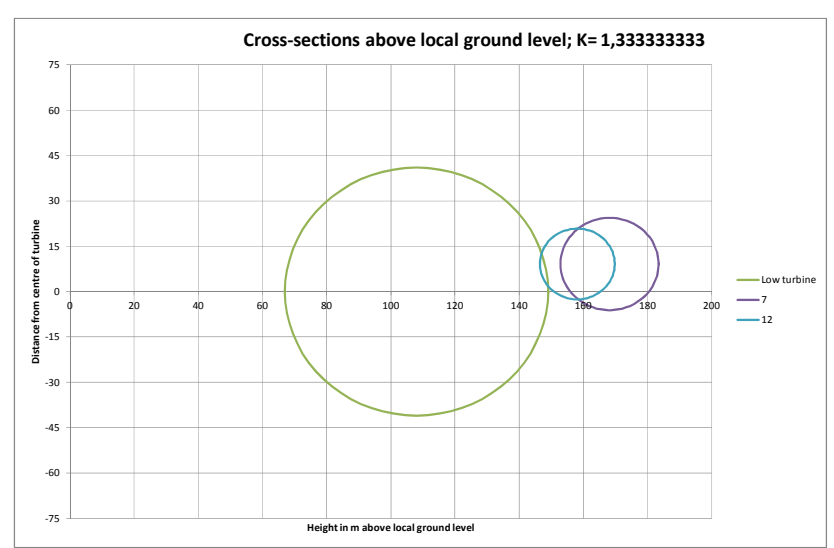

Fig. 6: Cross-section of a low wind turbine located below two microwave links (at 7 and $12 \mathrm{GHz}$, with antennas on the same towers) at the location of the turbine for a standard atmosphere. 


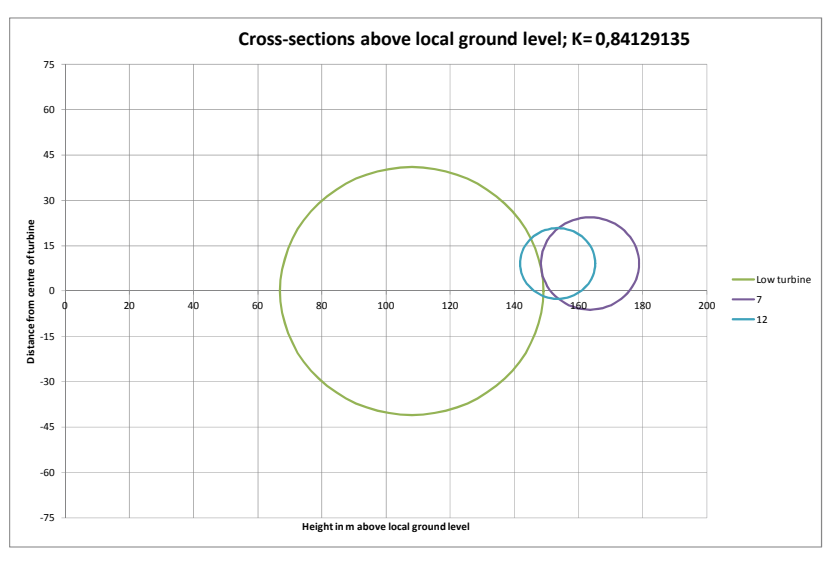

Fig. 7: Cross-section of a low wind turbine located below two microwave links (at 7 and $12 \mathrm{GHz}$, with antennas on the same towers) at the location of the turbine for a nonstandard atmosphere $(K=0.84129135)$.

For the non-standard atmosphere, the value of $K$ is, in a very good approximation, for a microwave link $\left(h<<R_{a}\right)$ and $\psi \cong 0$, given by:

$$
K=R_{e q} / R_{a} \cong 1 /\left[1+R_{a} / n\left(\frac{d n}{d h}\right)\right]
$$

This implies that we need to know the derivative of the refraction index with the height above the local ground. We can easily obtain this derivative from (1):

$$
\begin{aligned}
& \frac{d n}{d h}=77.6 * 10^{-6}\left[\frac{d p}{d h}+(4810 / T-0.072) \frac{d e}{d h}\right. \\
& \left.-(p-0.072 e+9620 e / T) / T \frac{d T}{d h}\right] / T
\end{aligned}
$$

or

$$
\frac{d n}{d h}=c t_{1} \frac{d p}{d h}+c t_{2} \frac{d e}{d h}+c t_{3} \frac{d T}{d h}=t_{1}+t_{2}+t_{3}
$$

\section{Discussion of the meteorological measurements}

The Royal Meteorological Institute of Belgium in Uccle, Brussels, is performing twice daily (until Nov. 2003) or three times a week (after Nov. 2003) measurements with a weather balloon, allowing to retrieve the vertical profile of all needed parameters like the temperature, pressure, and humidity in function of altitude. Here, we use only the measurements by sensors carried by these weather balloons, and not from ground-based devices. The extreme values recorded in the time period 1968-2016 are summarised in Table 1.
Table 1: Values of the maxima and minima of the important meteorological parameters at low altitude (the subscript $s$ denotes surface, or $h=0$ ) at Uccle, Brussels.

\begin{tabular}{|l|r|r|r|}
\hline Variable & Dimension & Minimum & Maximum \\
\hline$T_{s}$ & $\mathrm{~K}$ & 256.050 & 307.150 \\
\hline$P_{s}$ & $\mathrm{hPa}$ & 946.000 & 1110.400 \\
\hline$e_{s}$ & $\mathrm{hPa}$ & 0.278 & 29.941 \\
\hline$d T / d h<200$ & $\mathrm{~K} / \mathrm{m}$ & -0.107 & 0.060 \\
\hline$d p / d h<200$ & $\mathrm{hPa} / \mathrm{m}$ & -0.403 & -0.107 \\
\hline$d e / d h<200$ & $\mathrm{hPa} / \mathrm{m}$ & -0.105 & 0.050 \\
\hline
\end{tabular}

\section{Determination of the extreme $K$ values}

The maximal value for $\mathrm{n}$ (or the refractivity $N=(n-1)^{*} 10^{6}$ ) is obviously found by using the maximal pressure values and the minimal temperature values:

$$
N_{\max }=77.6 / T_{\min }\left[p_{\max }-\left(0.072+4810 / T_{\min }\right) e_{\max }\right]
$$

The minimal value is analogously obtained by:

$$
N_{\min }=77.6 / T_{\max }\left[p_{\min }-\left(0.072+4810 / T_{\max }\right) e_{\min }\right]
$$

The acquired minimal and maximal values for the refractivity are respectively 77.7 and 506.3. They are really worst cases, since in fact $N$ should be computed for every measurement, since the maximal partial pressure of the water vapour does not occur at the same time as the maximal total pressure, for instance. For the derivative, it is more complicated, since some values can be negative (apparently only $d p / d h$ is always negative, what is expected from the laws of physics of gasses). We have to deal with 3 terms. The minimal and maximal values of the coefficient of the first term $c t_{1}$ are $0.253 * 10^{-6}$ and $0.303 * 10^{-6}[1 / \mathrm{hPa}]$. When including the derivative of the total atmospheric pressure, the extreme value of the first term $t_{l}$ varies between $-0.122 * 10^{-6}$ and $-0.027 * 10^{-6}[1 / \mathrm{m}]$. The coefficient of the second term varies between

$$
c t_{2, \max }=77.6^{*} 10^{-6}\left(4810 / T_{\min }-0.072\right) / T_{\min }
$$

and

$$
c t_{2, \min }=77.6 * 10^{-6}\left(4810 / T_{\max }-0.072\right) / T_{\max }
$$

The numerical values for the minimum and the maximum of this coefficient of the second term are hence $3.938^{*} 10^{-6}$ and $5.671 * 10^{-6}[1 / \mathrm{hPa}]$. When we now include the derivative of the partial pressure of the vapour content, we obtain limiting values for the second term $t_{2}$ between $0.595 * 10^{-6}$ and $0.284 * 10^{-6}[1 / \mathrm{m}]$. The third term $t_{3}$ is the most complex. the coefficient varies between

$$
\begin{aligned}
& c t_{3, \text { min }}=-77.6 * 10^{-6}\left[p_{\text {max }}+\left(9620 / T_{\min }-0.072\right) e_{\max }\right] / T_{\text {min }}^{2} \\
& \text { and } \\
& c t_{3, \text { max }}=-77.6 * 10^{-6}\left[p_{\text {min }}+\left(9620 / T_{\max }-0.072\right) e_{\min }\right] / T_{\max }^{2}
\end{aligned}
$$

So, the coefficient of the third term varies between $-2.643 * 10^{-6}$ and $-0.785^{*} 10^{-6}[1 / \mathrm{K}]$. Taking into account the derivative of the temperature, we obtain for the third term the extreme values of $-0.159 * 10^{-6}$ and $0.283 * 10^{-6}$ $[1 / \mathrm{m}]$. 
This yields us finally the extreme values for the derivative of the refraction index $d n / d h$. This derivative varies between $-0.876^{*} 10^{-6}$ and $0.539^{*} 10^{-6}[1 / \mathrm{m}]$. Note that the value of $d n / d h$ for the standard atmosphere is $-0.039 * 10^{-6}$ $[1 / \mathrm{m}]$.

Now, we have to fill all the extreme values for $n$ and $d n / d h$ in (3) to find the extreme values for the $K$ factor.

$$
K_{\max } \cong 1 /\left[1+R_{a} / n_{\min }\left(\frac{d n}{d h}\right)_{\min }\right]
$$

and

$$
K_{\text {min }} \cong 1 /\left[1+R_{a} / n_{\text {min }}\left(\frac{d n}{d h}\right)_{\text {max }}\right]
$$

Note that to obtain the largest $K$ value, we have to use the smallest denominator. Therefore, since the minimum of $d n / d h$ is negative, we have to divide $d n / d h$ by the smallest value of $n$ (but it does not matter much since $\mathrm{n}$ is close to one). This is also the case for the maximal value. The extreme values for $K$ are then -0.218 and 0.225 . So in extreme conditions, $K$ can start at 0.225 (bending the wave away from the earth; i.e. substandard atmosphere), then gradually increases over the free space situation with $K=1$ to the $4 / 3$ of the standard atmosphere over the super standard atmosphere to $K=+\infty$ (in that case the wave follows the earth). The $K$ becomes negative (ducting) and can go up to the value of -0.218 .

\section{Application to the microwave links}

Taking into account those extreme $K$ values, it is now obvious that the good working of the second microwave link could not be guaranteed in all circumstances. Indeed, from the cross-section (Fig. 8), we notice that the blades could nearly completely shadow the link (the blades do not cover the diameter of the first Fresnel ellipsoid (15.28 m) completely).

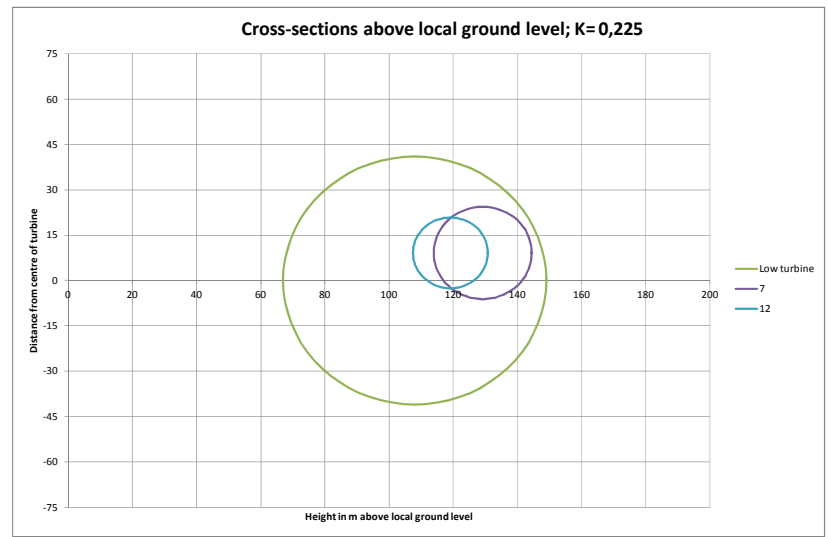

Fig. 8: Cross-section of a low wind turbine located below two microwave links (at 7 and $12 \mathrm{GHz}$, with antennas on the same towers) at the location of the turbine for the worst case atmosphere $(K=0.225)$.

The first microwave link however looks much better (Fig. 9) now: the wave will never deviate as much as in Fig. 5.

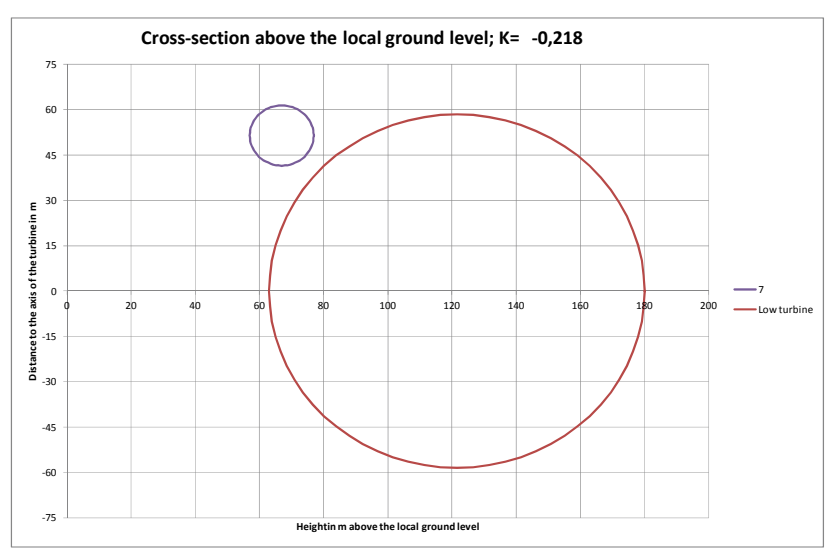

Fig. 9: Cross-section of a high wind turbine located above a microwave link at the location of the turbine for the worst case atmosphere $(K=-0.218)$

\section{Conclusions}

We have derived formulas to obtain the worst case trajectories (from $K$ values) for terrestrial microwave links based on (partial derivatives) of boundary layer meteorological data available above the location of the Royal Meteorological Institute of Belgium in Uccle (Belgium). This might help in trying to implement green energy, generated by wind turbines, without influencing actual microwave links. A statistical analysis with the same data might be possible, but therefore, we should first verify the statistics of the data, which might not be Gaussian.

\section{Acknowledgements}

We are greatly indebted EDF-Luminus for the examples of links.

\section{References}

1. Radio Advisory board of Canada, "Technical Information and Coordination process Between Wind Turbines and Radiocommunication and Radar systems". Can be downloaded (8/1/2018) from: https://www.rabc-cccr.ca/about/publications/windturbines-radio-radar

2. ITU-R, recommendation P.453-11, "The radio refractive index: its formula and refractivity data", 07/2015

3. Enrique Fernandez and Marc Mathieu, "Les faisceaux Hertziens analogiques et numériques", Bordas/CNETENST, Paris, 1991, ISBN 2-04-018737-5

4. https://www2.jpl.nasa.gov/srtm/

5. ITU-R, recommendation P.526-13, "Propagation by diffraction", 11/2013 